\title{
Synthesis of Unmodified Oligonucleotides
}

\section{INTRODUCTION}

B ecause of the significant progress achieved in the chemical synthesis of oligonucleotides during the past four decades, synthetic oligonucleotides have become readily available and have fueled the biotechnology revolution that has irreversibly changed biomedical research and the pharmaceutical industry. For example, without the ability to rapidly and efficiently synthesize DNA oligonucleotides, the development of the polymerase chain reaction (PCR) and its multiple applications would have been difficult, if not impossible, because this technology is completely dependent upon the use of DNA primers. Similarly, the availability of synthetic oligonucleotides has been instrumental in the development of automated DNA sequencing, which is also dependent on the use of DNA primers. An important biological application of synthetic oligonucleotides relates to site-specific mutagenesis of genes. Mutagenesis of this type has been utilized to study protein structure-function relationships, and to alter the therapeutic spectrum of pharmaceutically active proteins. Synthetic oligonucleotides have also found application as diagnostic hybridization probes and, more recently, in the use of DNA chips for highthroughput detection of genetic diseases and identification of genomic single-nucleotide polymorphisms. Furthermore, the availability of synthetic RNA oligonucleotides has led to the preparation of ribozymes, which are RNAs with catalytic activity. In this regard, it is being speculated that RNA may have been the primary self-replicating molecule from which life originated. Collectively, the multiple biomedical applications of synthetic DNA and RNA oligonucleotides are a direct measure of the colossal impact the methods for rapid and efficient preparation of these biomolecules have had on the life sciences.

Like Chapter 2, this chapter is composed of a number of overviews that provide investigators with a fundamental knowledge of solid-phase oligonucleotide synthesis, as well as a number of step-by-step protocols delineating the preparation of various oligonucleotides from suitably protected nucleoside phosphoramidite or $\mathrm{H}$-phosphonate derivatives.

UNIT 3.1 browses through the physical and chemical properties of liquid-phase and solidphase supports that have been developed over the years, and compares their suitability for oligonucleotide synthesis. The unit also examines the properties of linkers for transient or permanent attachment of properly protected nucleosides to the derivatized support of interest. Conditions for the release of synthetic oligonucleotides from specific supports have been discussed in detail. A step-by-step protocol for the attachment of nucleosides to commonly functionalized supports via either a succinyl or a hydroquinone- $O, O^{\prime}$-diacetyl linker is provided in UNIT 3.2.

Solid-phase synthesis of oligonucleotides is governed by a number of parameters. For example, UNIT 3.3 reviews the mechanism of activation of deoxyribonucleoside phosphoramidites, the type of activators required for such activation, and the consequences of an activator's acidity on the production of synthetic oligonucleotides. Steric factors affecting the condensation rates of deoxyribonucleoside phosphoramidites and the significance of "capping" and oxidation reactions in the chemical synthesis of oligonucleotides, along with oligonucleotide deprotection strategies, are discussed at length in the unit. Finally, UNIT 3.3 deals with the application of dinucleotide and trinucleotide phosphoramidites

Contributed by Serge L. Beaucage

Current Protocols in Nucleic Acid Chemistry (2004) 3.0.1-3.0.2

Copyright $(C) 2004$ by John Wiley \& Sons, Inc.

Synthesis of Unmodified

Oligonucleotides

3.0.1

Supplement 18 
as alternatives to monomeric deoxyribonucleoside phosphoramidites in the synthesis of DNA oligonucleotides.

Additional synthetic strategies and parameters related to the synthesis of oligodeoxyriboand oligoribonucleotides according to the $H$-phosphonate method are reviewed in UNIT 3.4; an emphasis on the preparation and activation of nucleoside $H$-phosphonate monoesters is maintained throughout the unit. This information accurately defines the scope and potential of the method for oligonucleotide synthesis.

The chemical synthesis of DNA oligonucleotides without nucleobase protection has been and is still an elusive goal. An overview of the strategies that have been developed so far to achieve this goal is presented in UNIT 3.10.

Strategies in the selection of $\mathrm{N}-, 5^{\prime}-O-$, and $2^{\prime}-O$-protecting groups for efficient synthesis of oligoribonucleotides according to the phosphoramidite approach are discussed in depth in UNIT 3.5. Critical synthesis parameters such as the type of support being used and selection of the optimal activator for ribonucleoside phosphoramidites, as well as oligonucleotide deprotection protocols, are also reviewed in the unit to provide a comprehensive account of RNA phosphoramidite chemistry. As a logical extension of UNIT 3.5, the solid-phase synthesis of oligoribonucleotides with $2^{\prime}$-O-tert-butyldimethylsilyl, $2^{\prime}$ $O$-(2-nitrobenzyloxymethyl), or $2^{\prime}-O$-[(triisopropylsilyl)oxy]methyl groups according to the phosphoramidite approach is delineated in UNITS 3.6-3.8, respectively, along with detailed methods for the deprotection of these oligonucleotides.

In future supplements, this chapter will also incorporate step-by-step protocols describing new methods for the synthesis of unmodified DNA oligonucleotides that employ either new phosphate-protecting groups or novel coupling procedures to accommodate specific nucleic acid applications. In this regard, the use of 3-( $N$-tert-butylcarboxamido)1-propyl and 4-oxopentyl groups for phosphate/thiophosphate protection in solid-phase DNA synthesis is presented in UNIT 3.9. These protecting groups may find application in large-scale preparations of therapeutic oligonucleotides. Likewise, oligoribonucleotide synthesis protocols comparing the properties of selected $2^{\prime}-O$-protecting groups, coupling methods, and deprotection conditions will be included in an effort to contribute the best nucleic acid tools to investigators pushing further the frontiers of knowledge.

Serge L. Beaucage

Introduction 